\title{
Minerals under extreme conditions: Heavy ion irradiations under high pressure and in-situ Raman spectroscopy
}

\author{
I. Tzifas 1*, K. Voss1, R. Böhler2,3, M. \\ Lang, B. Merk1, D. Severin1, E.M. \\ Toimil-Morales1, C. Trautmann1,5
}

1GSI Helmholtz Center for Heavy Ion Research, Planckstr. 1, 64291 Darmstadt, Germany

(*correspendence: i.tzifas@gsi.de)

2 Department of Nuclear Engineering, University of Tennessee, Knoxville, TN 37996-2200, USA

3 Neutron Scattering Division, Oak Ridge National

Laboratory, Oak Ridge, TN, USA

${ }_{4}$ Geophysical Laboratory, Carnegie Institution for Sciences, Washington DC 20015, USA

5Technische Universität Darmstadt, Alarich-Weiss-Str. 2, 64287 Darmstadt, Germany

\begin{abstract}
Driven by fundamental questions related to Earth and materials sciences, as well as physics, chemistry highpressure research has been advancing rapidly over the past few decades. The coupling of high-pressure technology with ion beams has opened up new possibilities to simulate radioactive decay events in compressed and heated minerals of Earth's interior or to modify thermodynamic pathways of unstable high-pressure materials such that they can be recovered to ambient conditions [1,2]. This approach is realized by injecting heavy ions of relativistic energies (e.g., $60 \mathrm{GeV} \mathrm{U}$ ions) into miniaturized samples that are pressurized within a diamond anvil cells (DAC). This presentation provides an overview of previous results and show new developments at the heavy-ion synchrotron (SIS18) of the GSI accelerator facility, related to the coupling of ion beams and pressure cells, including the installation of in situ characterization capabilities by means of Raman spectroscopy. This requires modifications to the existing approach to avoid beam-induced coloration of the diamond anvils.
\end{abstract}

[1] Lang, M., F. Zhang, J. Zhang, J. Wang, B. Schuster, C. Trautmann, R. Neumann, U. Becker, and Ewing (2009) Nature Materials, 8(10), 793-797.

[2] Lang, M., Lian, J., Zhang, F., Hendriks, B.W., Trautmann, C., Neumann, R. and Ewing, R.C., 2008. Earth and Planetary Science Letters, 2008. 274(3-4): p. 355-358. 